sometimes given rather haphazardly (for example there is no mention of Newman in connection with the charged Kerr solution, nor of Bondi in relation to the mass carried by gravitational waves). Finally, the absence of any discussion of 'mixmaster' singularities or of any details of the Belinskii - Lifshitz - Khalatnikov work seems to me to be a serious omission.

I would like to emphasise that these criticisms are basically minor ones in view of the formidable and impressive task undertaken by the authors. They have succeeded admirably and their work is undoubtedly a landmark in the presentation of modern gravitational theory.

The question of where the universe comes from and where it is going cannot fail to be of interest to everyone. There is rather little in science which directly bears on this question, but if anything seems to, it is surely the subject matter so powerfully presented in this book.

\section{Roger Penrose}

\section{Food manufacture}

Biology and the Food Industry. By H. R. Barnell. Pp. 59. (The Institute of Biology's Studies in Biology, No. 45.) (Arnold: London, February 1974.) $£ 1.50$ boards; 75 p paper.

THE story is told of an Oxford undergraduate who obtained consistently good grades for his essays by copying from the Encyclopedia Britannica which his tutor had never read. To help "students at school, college and university . . . to keep abreast of recent trends and know where the most significant developments are taking place", the Institute of Biology sponsors the preparation of a series of booklets on selected aspects of biology to supplement the more substantial but inevitably finite treatment of biology which a textbook could be expected to provide. The late Dr Rex Barnell has written such a booklet about the food industry. In it he has systematically described such salient features of manufacturing processes and methods of food preservation as could be expected within the constraint of 59 pages, bearing in mind that he had also to touch on food composition, nutrition and the future. $\mathrm{He}$ has performed his task well and both pupils and their teachers will be grateful whether or not there may be some readers who feel a sneaking sympathy for the Oxford tutor.

Here will be found the distinction between drying and dehydration, a definition of a convenience food, description of pasteurisation, canning, chilling, freezing, irradiation and four lines about pickling. Ten pages suffice for milling, baking and margarine making. Vegetable meats, leaf protein and micro organisms grown on hydrocarbons get seven pages. It is all very neatly done. The section devoted to nutrition and food quality sensibly draws attention to the contribution manufacturers make to an adequate diet by making their commodities readily available. In the breathless pace, however, figures for energy value seem to have slipped out of the table of "recommended intakes". The book ends on a high note with the promise that "it is by no means impossible that if groups of biological, physical and chemical research workers concentrate on the problems . . . virtually unlimited supplies of food ... may be truly synthesised ... from constituents of the earth's atmosphere and crust". Well, perhaps they may.

\section{MAGNUS PyKe}

\section{Reactions at electrodes}

The Chemistry of Electrode Processes. By Hana Fried. Pp. $x+225$. (Academic: London and New York, January, 1974.) $£ 4.90 ; \$ 13.75$.

RECENTLY, a number of books have appeared based on the study of electrode reactions or electrodics. According to the author, this monograph is designed as "an introduction to electrodics" for "the advanced student or the qualified chemist" who possesses "no familiarity with electrochemistry". The author has succeeded in achieving her goal and has produced a concise and very readable book. Every section is prefaced by a clear statement of the question to be answered and derivations have been kept simple by mixing rigorous treatments with comparisons and analogies. The SI unit system and IUPAC conventions have been adhered to throughout. Furthermore, the book contains a balance between theory, experimental details and areas of application.

The book is divided into six chapters; the first two are brief and introduce the area of interest, the fundamental concepts and definitions. The third chapter deals with the kinetics of electrode reactions by developing the relationships between current, potential and time for both reversible and irreversible electron transfer processes. Moreover, irreversible processes caused by slow chemical reactions preceding charge transfer and reactant adsorption are also considered. Finally, the influence of cell impedance on the current-potential behaviour is discussed. The fourth chapter which completes the theoretical part of the monograph is devoted to the electrode-solution interphase. Various models of the double layer are evalu- ated and the modern model which offers the best fit to the experimental results is described. The topic of specific adsorption is also examined because of its effects on the double layer. The final section discusses how electrode reactions are influenced by the electrode-solution interphase.

The fifth chapter introduces the methodology of electrode reactions. Included in this section are descriptions of cells, electrodes and apparatus; equilibrium measurements of capacitance and impedance; voltammetric methods; and potential, current and charge step techniques together with their limits of applicability. The chapter concludes by mentioning the techniques of reflection spectroscopy and transmission spectroscopy at optically transparent electrodes. One drawback to this chapter, in particular, is the lack of references to the literature. In fact, the bibliography contains only a selection of books that the author "found useful" and omits all references to recent reviews and original contributions.

The final chapter offers little more than an indication of the number of areas where electrode reactions are involved, for example, corrosion, electroplating, and fuel cells. This broad survey was too ambitious and the author would have been more successful in illustrating the applications of electrodics by examining, in detail, a limited number of solutions to problems related to the areas mentioned. Such an approach would have supplemented the material of chapter 5 and, by involving the reader in the problemsolving prozess, would have further familiarised him with the study of electrode processes.

\section{M. ELSON}

\section{Cultured plants}

Plant Tissue and Cell Culture. Edited by H. E. Street. Pp. viii +503 . (Botanical Monographs, Vol. 11). (Blackwell Scientific: Oxford and London, 1973.) $£ 12.50$.

Although plant tissues were first cultured in vitro 35 years ago, the realisation of their potential has always seemed to remain tantalisingly in the future. The technical problems of initiating and maintaining cultures of all but a few particularly favourable plants have until quite recently seemed insuperable obstacles to achieving the ready answers to physiological questions that the tissue and cell culture approach seems to offer. This excellent book should completely dispel that notion. Professor Street has a knowledge and experience of the culture of plant tissues, and particularly plant cells, that is second to none, and with his cust- 